\title{
Numerical analysis and optimum design for the vacuum jacket valve
}

\author{
Rock-Won Jeon ${ }^{1}$, Si-Pom Kim ${ }^{2}$, , Jae-Hoon Lee ${ }^{3}$ \\ 1, 2, 3 Department of Mechanical Engineering, Dong-A University,Hadan 2-dong, Saha-gu, Busan, \\ South Korea
}

\author{
Index Terms \\ Vacuum-Jacket Valve \\ Heat Transfer \\ Numerical Analysis \\ Design of Experiment \\ Optimization
}

Received: 19 October 2014

Accepted: 20 July 2015

Published: 22 February 2016

\begin{abstract}
Cryogenic vacuum jacket valves are used to supply smooth cryogenic gas and minimize generation of BOG (Boil-off Gas) by intercepting intrusion of heat being generated inside valves in liquefied flow supply of cryogenic equipment through the use of a vacuum system. This research focused on the interception of heat exchange with the outside environment. It also considered heat transfer properties on changes in pressure of a vacuum part, a radius of a jacket, and jacket length, which can reduce heat exchange for effective heat transmission control. Therefore, by using the design of the experiment, one of the optimization strategies for the design of cryogenic vacuum jacket valve, we selected the combination of main design factors. For qualifying the design technology, we compared and analyzed the results using numerical analysis.
\end{abstract}

(C) 2016 TAF Publishing. All rights reserved.

\section{INTRODUCTION}

A cryogenic vacuum jacketed valve is used to smoothly supply ultra-low temperature gas and minimize boil-off gas (BOG) by blocking the penetration of the heat generated by the liquid flow supply of ultra-low temperature equipment using a vacuum system [1], [3]. In addition, as a result of various environmental regulations associated with global warming, the valve is usually applied to equipment that uses clean fuel, which generates a smaller amount of pollutants, and demand for this valve has been increasing [4].

Recently, with the rapid development in its industry, there has been continuous research on the cryogenic valve, and a trend of increasing performance owing to rapid

\footnotetext{
*Corresponding author: Si-Pom Kim

E-mail:spkim@dau.ac.kr
}

development in the fields of machinery, shipbuilding, semiconductors, displays, and aerospace, in addition to user demand. However, there is insufficient technological development and research on ultra-low temperature application equipment for vacuum insulation [5].

The peculiarity of having its fluid at an ultra-low temperature as resulted in the cryogenic valve is being prone to serious problems during manufacturing, installation, and operation in pipelines, such as loss of function because of stiffening of packing or gas leakage. Even when highly ductile materials are applied in order to prevent gas leakage at ultra-low temperatures, it is extremely difficult to completely block gas leakage in gaps formed near gaskets as a result of stiffening. Consequently, recent researches have actively been performed on controlling the internal temperature by increasing the structure or length of the hood to minimize this problem [6]. 
This study focused on decreasing the influx of heat to the interior of the jacket by maintaining a vacuum after piping is installed outside of the stem for efficient heat transfer at ultra-low temperatures. This is unlike previous studies that attempted to reduce problems generated by temperature decline. A method that uses pressure change inside the jacket is effective because it can reduce the increase in material cost and economic loss that would occur as a result of changes in hood structure or length, and simultaneously solve the problems that occur in existing cryogenic valves. Therefore, for an effective research, we deducted an optimal design factor that affects heat transfer in the vacuum jacketed valve by applying an experimental design, which is an optimization method.

\section{NUMERICAL ANALYSIS}

For normal operation of the valve at ultra-low temperatures $\left(-196^{\circ} \mathrm{C}\right)$, it is important to control the heat transfer from the internal low-temperature fluid in the liquefied natural gas (LNG) region to the upper portion of the packing. Consequently, in this study, heat transfer was controlled by adding a jacket near the valve stem, and applying a very low pressure to the space between the stem and jacket. In addition, several design variables were selected for effective control of heat transfer in the vacuum jacketed valve. A total of eight design variables, including jacket thickness, jacket length, stem thickness, and material emissivity, were selected. These variables were then used in numerical analysis. Conduction occurs in the interior of the valve, as well as convective heat transfer between the external atmosphere and jacket surface.

Therefore, this study evaluated the effect of the selected design variables on heat transfer characteristics through a consolidated analysis accounting for the relationship between convection to the external atmosphere and internal conduction.

\section{A. Theory}

Air, which is the fluid in the external flow region, flows along the surface of the jacket, and the internal and external temperatures are different. As a result, convection occurs between the fluid and solid surface owing to the liquid's motion. The equation applied to this phenomenon is Newton's law of cooling, which is shown in Eq. 1.

$Q=h A\left(T_{s}-T_{\infty}\right) \quad[W]$
In addition, numerical analysis was performed using Shear Stress Transport (SST) turbulence model because turbulent flow was expected to occur in the external atmosphere region and a local area in the valve body. The model was naturally switched into the $\mathrm{k}-\omega$ turbulence model for the turbulent flow near the wall surface and the $k$ - $\varepsilon$ turbulence model for the turbulent flow in the free flow region. The equation applied was the governing equation for the turbulent kinetic energy $k$ and turbulence frequency $\omega$, in the form of Eq. 2-3.

$$
\begin{aligned}
& \frac{\partial}{\partial t}(\rho k)+\frac{\partial}{\partial x_{j}}\left[\rho\left(u_{j}-u_{s j}\right) k-\left(\mu+\frac{\mu_{i}}{\sigma_{k}}\right) \frac{\partial k}{\partial x_{j}}\right] \\
& =\mu_{i}\left(P+P_{B}\right)-\rho \beta \omega k-\frac{2}{3}\left(\mu_{t} \frac{\partial u_{i}}{\partial x_{i}}+\rho k\right) \frac{\partial u_{k}}{\partial x_{k}}
\end{aligned}
$$

$$
\begin{aligned}
& \frac{\partial}{\partial t}(\rho \omega)+\frac{\partial}{\partial x_{j}}\left[\rho\left(u_{j}-u_{s i}\right) \omega-\left(\mu+\frac{\mu_{t}}{\sigma_{\omega}}\right) \frac{\partial \omega}{\partial x_{j}}\right] \\
& =\frac{\gamma}{\nu_{i}}\left[\mu_{i} P-\frac{2}{3}\left(\mu_{t} \frac{\partial u_{i}}{\partial x_{j}}+\rho k\right) \frac{\partial u_{k}}{\partial x_{k}}\right]+\frac{\delta}{\nu_{t}} \mu_{i} P_{B} \\
& -\beta \rho \omega^{2}+2\left(1-F_{1}\right) \rho \frac{1}{\sigma_{\omega t}} \frac{1}{\omega} \frac{\partial k}{\partial x_{j}} \frac{\partial \omega}{\partial x_{j}}
\end{aligned}
$$

\section{B. Design of Experiments}

To design the vacuum jacketed valve, we selected basic design factors using experimental design, which is one method of design optimization, and selected meaningful design variables using an orthogonal array. For the experimental design, an L18 orthogonal array was made for eight control factors, with three levels each. This method allowed us to reduce the number of experiments from a total of $2 \times 37=4374$ to 18 , and to deduce the optimal factor from just those 18 experiments. Consequently, analysis of variance (ANOVA) was conducted based on experimental design, and the optimal level was determined following calculation of each factor's level of contribution. Table 1 presents the control factors and levels.

TABLE1

ORTHOGONAL ARRAYS

\begin{tabular}{ccccc}
\hline \hline & Control factor & \multicolumn{3}{c}{ Level } \\
\hline A & Shape of jacket & 1 & 2 & - \\
B & Thickness of jacket & 1 & 2 & 3 \\
C & Diameter of inner jacket & 1 & 2 & 3 \\
D & Length of plate & 1 & 2 & 3 \\
E & Thickness of pipe & 1 & 2 & 3 \\
\hline \hline
\end{tabular}




\begin{tabular}{ccccc}
\hline \hline $\mathrm{F}$ & Thickness of packing & 1 & 2 & 3 \\
$\mathrm{G}$ & Emissivity & 1 & 2 & 3 \\
$\mathrm{H}$ & Insulating materials & 1 & 2 & 3 \\
\hline \hline Numerical & analysis was conducted & \multicolumn{2}{c}{ using }
\end{tabular}
experimental design, and its results were analyzed using ANOVA. The results of the analysis showed that control factors $\mathrm{A}, \mathrm{B}$, and $\mathrm{C}$ had large differences for each level, but not in terms of other aspects. In other words, the factors that influence the heat transfer in the vacuum jacketed valve are thought to be the jacket's thickness, inner diameter, and length. The optimal levels deducted based on this assumption are combinations of A2, B3, C3, D3, E2, F2, G2, and H3. ANOVA results can be found in Table 2 .

\section{Analysis Model}

The vacuum jacketed valve that is the subject of this study is mainly composed of a body, stem, and jacket, as depicted in Fig. 1. The external flow region was set based on the numerical analysis model to account for the effect of the external atmosphere temperature, which is an environmental factor. This setting was for the application of the operating conditions of the vacuum jacketed valve.

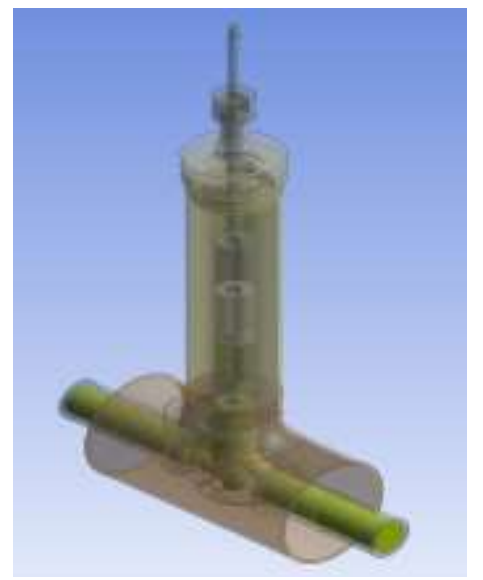

Fig. 1. Analysis model

The numerical analysis systems used in this study included fluid flow, steady-state thermal, and transient thermal analyses (ANSYS Workbench V14.0), and heat transfer analysis was performed to review the optimization factors. The transient thermal analysis was used for numerical analysis on the key factors to be examined.

The steady-state thermal analysis was used to apply the initial temperature condition by obtaining the temperatures of each system or component with respect to thermal loads. The transient thermal analysis was used to analyze several heat-related items including heat distribution with respect to time. Through this analysis, we reviewed heat distribution with respect to changes in design variables.

For effective numerical analysis, we simplified the model of interest, generating a 3D finite element model comprising nodes and elements. Because the internal and external forms change rapidly, an unstructured grid was used to generate a dense grid for more accurate results.

\section{Analysis Results}

Numerical analysis on the vacuum jacketed valve was implemented using experimental design, and its results were analyzed using ANOVA. The numerical analysis results corresponding to the deducted optimal levels are shown in Fig. 2-3.

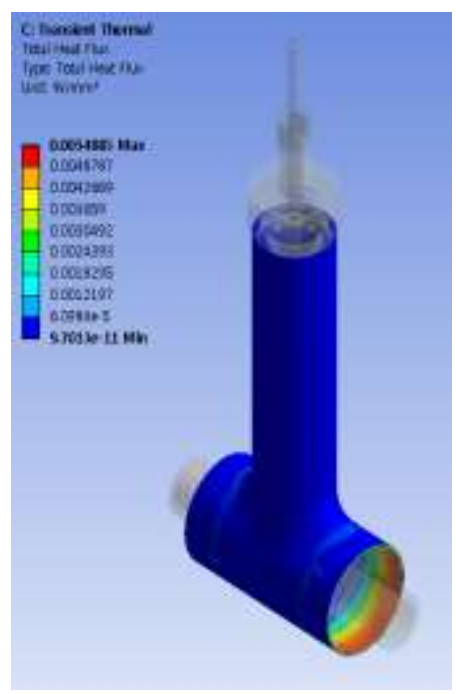

(a) Heat flux

Fig. 2. Thermal analysis results

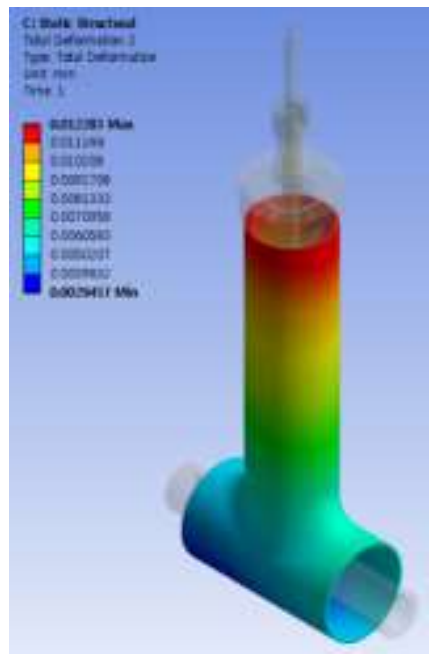

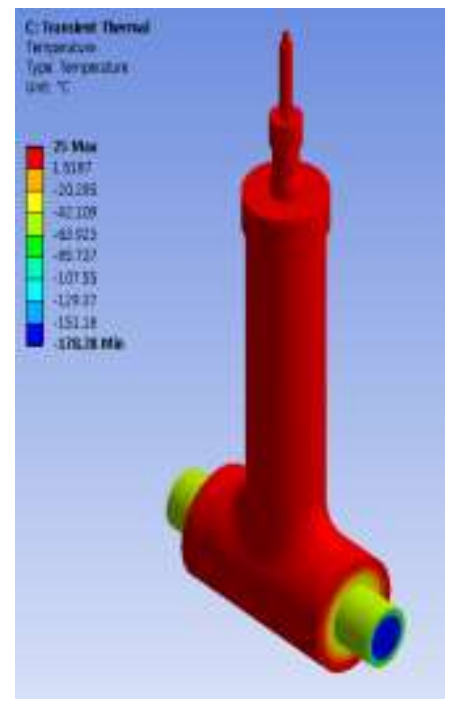

(b) Temperature

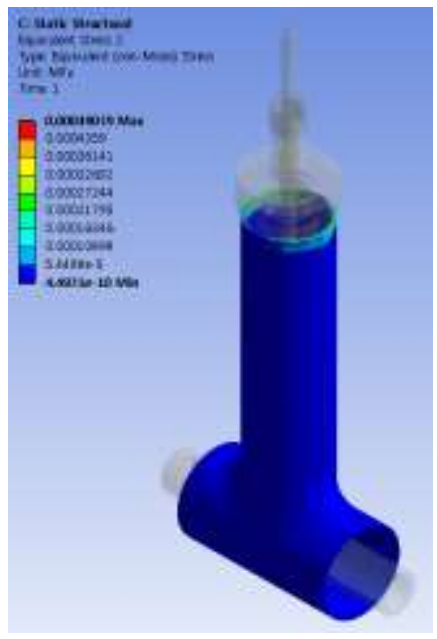


(a) Deformation

(b) Stress

Fig. 3. Thermal-structural analysis results

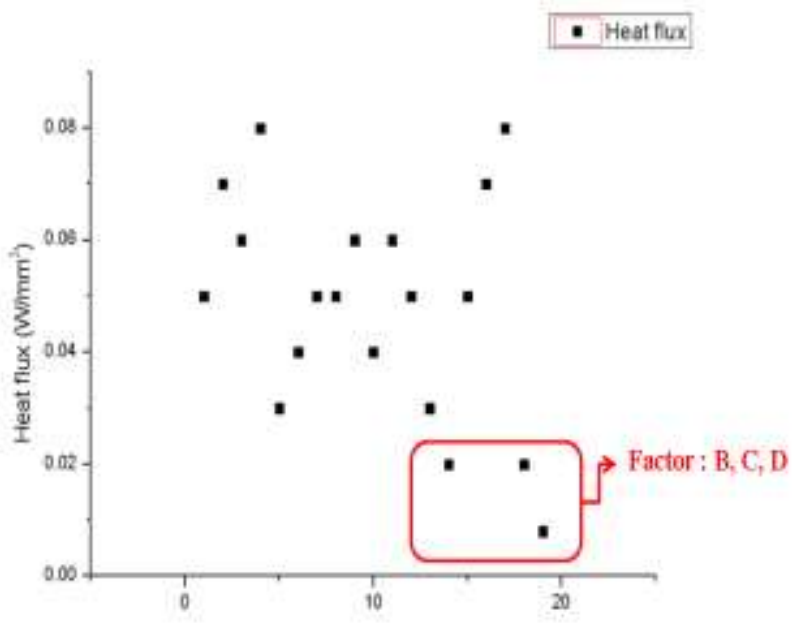

Fig. 4. Heat flux variations for DOE.

TABLE 2

ANALYSIS OF VARIANCES

\begin{tabular}{cccccc}
\hline $\mathbf{F}$ & S/N & $\boldsymbol{\beta}$ & $\mathbf{F}$ & S/N & $\boldsymbol{\beta}$ \\
\hline A1 & 2.12 & 213.7 & E1 & 12.53 & 562.55 \\
\hline A2 & 3.35 & 275.3 & E2 & 14.84 & 581.38 \\
\hline & & & E3 & 15.72 & 605.32 \\
\hline B1 & 10.55 & 437.8 & F1 & 7.91 & 353.49 \\
\hline B2 & 11.82 & 475.4 & F2 & 8.15 & 388.55 \\
\hline B3 & 13.75 & 528.2 & F3 & 9.73 & 423.17 \\
\hline & & & & & \\
\hline C1 & 12.63 & 447.3 & G1 & 3.68 & 287.52 \\
\hline C2 & 13.24 & 496.2 & G2 & 4.22 & 312.68 \\
\hline C3 & 14.97 & 508.7 & G3 & 3.51 & 293.84 \\
\hline & & & & & \\
\hline D1 & 8.9 & 431.4 & H1 & 5.14 & 395.21 \\
\hline D2 & 8.9 & 435.5 & H2 & 5.08 & 398.35 \\
\hline D3 & 8.8 & 465.8 & H3 & 7.85 & 413.87 \\
\hline & & & & & \\
\hline
\end{tabular}

The results show that for greater jacket thicknesses and diameters, the temperature of the upper portion of the valve is maintained closer to room temperature. It can be seen that the increase in the jacket's surface is considerably dominant in heat transfer, and we believe that studies on the jacket's surface area and optimal design of its form are crucial for normal operation of the jacketed valve at ultra-low temperatures.

\section{CONCLUSION}

This study performed numerical analysis on the effect of key design factors on the heat transfer of the vacuum jacketed valve at ultra-low temperatures. The analysis, which was conducted by applying an optimization method, provided the following results.

(1) A method of experimental design, an optimization method, was applied for efficient numerical analysis, and we reviewed the degree of effect on the key design factors by analyzing the results.

(2) ANOVA results show that the optimal levels of the control factors chosen in this study are combinations of A2, B3, C3, D3, E2, F2, G2, and H3.

(3) In particular, we believe that the jacket's thickness, inner diameter, and length (i.e., the jacket's surface area) have a significant effect on maintaining the temperature of the upper portion of the valve.

(4) The results of the numerical analysis for the optimal level indicate that the influx of heat from the outside decreased by about $7 \%$ compared to the initial model.

\section{ACKNOWLEDGEMENT}

This work was supported by the KEIT(Korea Evaluation Institute of Industrial Technology) which is the industrial innovation program (No. 2014-0712).

\section{REFERENCES}

[1] H. S. Witsenhausen, "A class of hybrid-state continuous time dynamic systems," IEEE Transactions on Automatic Control, vol. 11, no. 2, pp. 161-167, 1966. DOI: 10.1109/TAC.1966.1098336

[2] M. S. Branicky, V. S. Borkar and S. K. Mitter, "A unified framework for hybrid control: Model and optimal control theory," Automatic Control, IEEE Transactions on, vol. 43, no. 1, pp. 31-45, 1998. DOI: $10.1109 / 9.654885$

[3] R. Alur, C. Courcoubetis, T. A. Henzinger and P. H. Ho, "Hybrid automata: An algorithmic approach to the specification and verification of hybrid systems, in Hybrid Systems," in Lecture Notes in Computer Science, New York, NY: Springer-Verlag, 1993.

[4] P. Antsaklis, "Special issue on hybrid systems: Theory and applications. A brief introduction to the theory and applications of hybrid systems," in Proc. of the IEEE, 2000. 
[5] F. Torrisi and A. Bemporad, "HYSDEL-A tool for generating computational hybrid models for analysis and synthesis problems," IEEE Transactions on Control Systems Technology, vol. 12, no. 2, pp. 235249., 2004. DOI: 10.1109/TCST.2004.824309

[6] I. Kresno, "Perancanganperangkat MATLAB untukekuivalensi model sistem hybrid", dissertation,
Bandung Institute of Technology, Bandung, Indonesia, 2005.

[7] H. Ye, A. N. Michel and L. Hou, "Stability theory for hybrid dynamical systems," IEEE Transactions on Automatic Control, vol. 43, no. 4, pp. 461-474,1998. DOI: $10.1109 / 9.664149$

- This article does not have any appendix. — 